\title{
(1) \\ Multilocular Cystic Mass Located in Epigastric Region and Right Area of the Abdomen in an old man
}

Fariborz Mansour-Ghanaei ${ }^{1, *}$, Alireza Gharibpoor ${ }^{1}$, Farahnaz Joukar ${ }^{1}$, Sara Mavaddati ${ }^{1}$

1. Guilan University of Medical Sciences, Gastrointestinal \& Liver Diseases Research Center (GLDRC), Rasht, Iran

\footnotetext{
* Corresponding Author:

Fariborz Mansour-Ghanaei, MD, AGAF Professor, Division of Gastroenterology and Hepatology, Gastrointestinal and Liver Diseases Research Center (GLDRC), Razi Hospital, Guilan University of Medical Sciences (GUMS), Sardarjangal Avenue, Rasht, Iran Postal Code: 41448-95655 Tel: +981333535116 Fax: + 981333534951 Email: ghanaei@gums.ac.ir
}

Received: 03 Mar. 2017 Accepted: 09 Jun. 2017

\author{
Please cite this paper as: \\ Mansour-Ghanaei Fa, Gharibpoor AR, Joukar F, Mavaddati S. Multilocular Cystic Mass \\ Located in Epigastric Region and Right Area of the Abdomen in an old man. Middle East \\ J Dig Dis 2017;9:176-177. DOI: 10.15171/mejdd.2017.71.
}

A 67-year-old man presented with generalized abdominal pain, abdominal distension, and inability of defecation and gas passage, which persisted for two months. The pain was constant, which was first appeared in the epigastric and right upper quadrant (RUQ) regions but gradually became generalized. The pain intensified by bending and eating food while alleviated by lying. The patient complained of constipation, weight loss, and loss of appetite. He also reported a few times of vomiting after eating. He had no diarrhea, fever, icterus, dysuria, hematuria, shortness of breath, and coughing. There was no blood in the stool. The patient's medical history revealed an appendectomy in 20 years earlier. He was a chronic smoker without any history of opium and alcohol consumption. In physical examination, he was pale and had bilateral temporal atrophy. His jugular vein pressure was normal. The abdomen was distended and asymmetric with an apparent palpable mass extending to the epigastric, RUQ, and right lower quadrant (RLQ) regions. A surgical scar was seen in McBruny's point, which must have been from the earlier appendectomy (figure 1). The bowel sounds were hypoactive in all quadrants. Laboratory results were as the following: white blood cell count: $10.6 \times 103 / \mu \mathrm{L}$, hemoglobin: $12.9 \mathrm{gr} / \mathrm{dL}$, platelet: $175 \times 10^{3} / \mu \mathrm{L}$, prothrombin time (PT): $12.5 \mathrm{sec}$, partial thromboplastin time (PTT): $32 \mathrm{sec}$, INR: 1, blood urea nitrogen $(\mathrm{BUN}): 31 \mathrm{mg} / \mathrm{dL}$, creatinine: $1.24 \mathrm{mg} / \mathrm{dL}$, aspartate aminotransferase (AST): 24U/L, alanine aminotransferase (ALT): 11U/, amylase: 101U/L, lipase: 45U/, CRP: $12.2 \mathrm{mgL}$, and normal levels of electrolytes and urine analysis.

The sonography of abdomen showed extraordinary large cystic masses extended to the epigastric, and right upper and lower quadrants. The computed tomography (CT) of the abdomen and pelvic also revealed a large $9 \mathrm{~cm} \times 16 \mathrm{~cm} \times 20 \mathrm{~cm}$ multilocular cystic mass located in epigastric region and right area of the abdomen (figure 2). The mass caused a displacement in abdominal internal organs. In order to resect the mass, the patient underwent an exploratory laparotomy. Upon the operation, a retroperitoneal cystic mass was observed and some speci-

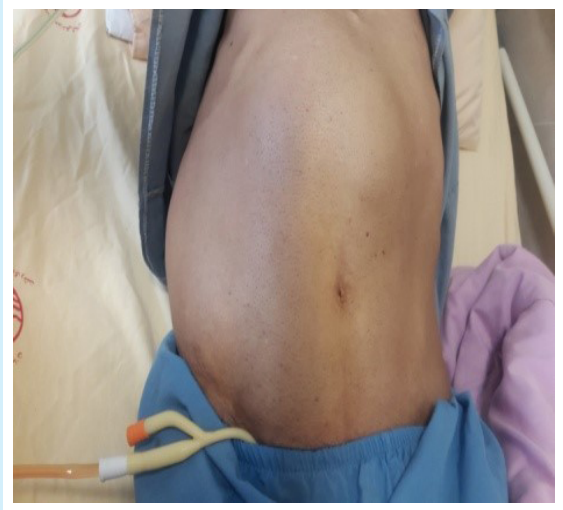

Fig. 1: Distended, asymmetric abdomen with an apparent palpable mass extending to the epigastric, and right and left upper quadrants as well as the surgical scar in McBurney's point.

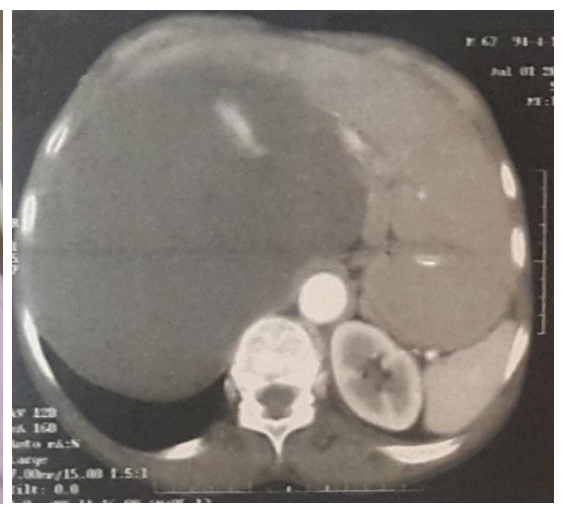

Fig. 2: A large $9 \mathrm{~cm} \times 16 \mathrm{~cm} \times 20 \mathrm{~cm}$ multilocular cystic mass located in epigastric region and right area of the abdomen. 


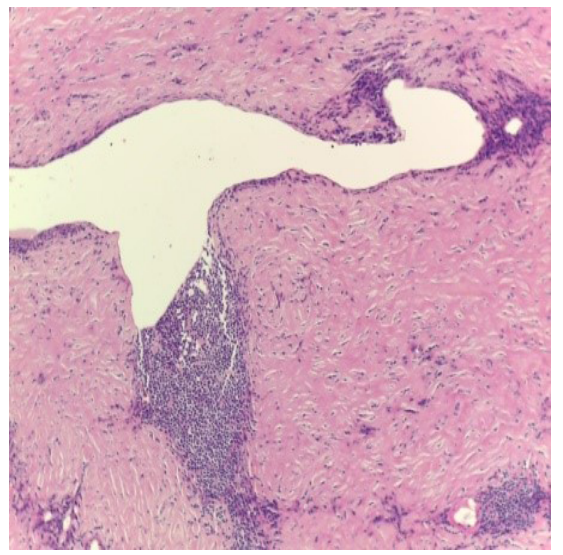

Fig. 3: Dilated cystic space lined by monolayer of bland endothelial cells and lymphoid aggregation in few areas.

mens were taken for further evaluations. Lymphangioma showed dilated cystic space lined by monolayer of bland endothelial cells. Also lymphoid aggregation was evident in few areas (figure 3). The mass was excised after removing its adhesions to the adjacent organs (figure 4).

What is your diagnosis?

Answer:

Retroperitoneal lymphangioma with multilocular cystic mass.

\section{DISCUSSION}

Variants, capillary, cavernous and cystic are three distinguishable variants of lymphangiomas. Cystic lymphangiomas, which are the most common ones, are characterized by dilated endothelium with smooth muscle, lymphoid tissue, and lymphocytes in the islands of the lumen within the cyst wall. ${ }^{1,2}$ In our case, the lesion consisted of dilated thinwalled lymphatic channels lined by attenuated, bland endothelial cells, which in some foci were surrounded by dense lymphocytic infiltrate. These tumors are symptomatic when become large. Left upper quadrant pain, gradually expanding abdominal mass, loss of appetite, nausea, and vomiting are the most common clinical manifestations. ${ }^{1}$ Enough enlarged mass, may compact or dislocate the urinary or bowel tract showing as chronic back or abdominal pain, or as feeling of fullness or distension ${ }^{3}$ as was seen in our patient. The clinical manifestation of abdominal distention and pressure on adjacent organs were owing to the fact of cystic expansion. Hemorrhage and inflammation of the cyst probably induce abdominal pain in accordance with leukocytosis. It seems that the probability of a viral infection opportunity was low because of negative bacterial and histological examinations. ${ }^{1}$ Since, preoperative diagnosis is rare, the result of histological examination of biopsy samples during exploratory laparoscopy or surgery is generally an assertive prognosis of cystic lymphangioma. ${ }^{2}$ Although, preopera-

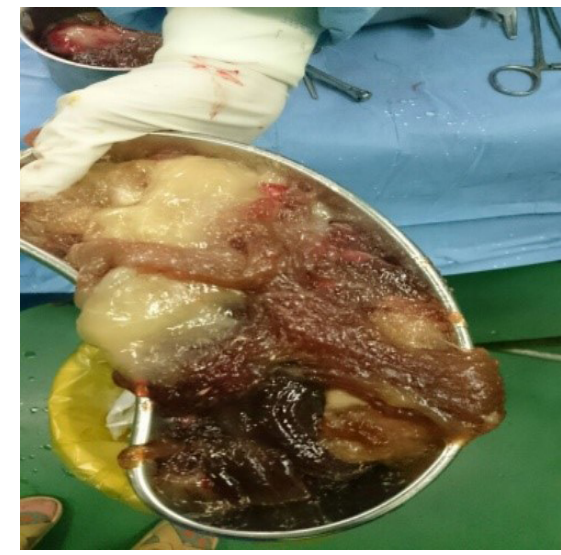

Fig. 4: Excised mass after removing its adhesions to the adjacent organs.

tive diagnosis of (lymphangioma) LA via lymphangiography may have an important impact, laboratory studies are not sufficient. Also, CT or nuclear magnetic resonance computed tomography (NMR) cannot give an apparent diagnosis. ${ }^{4}$ Because, invasion to vital structures and lifethreatening complications may develop subsequent to its growth, a considerable therapeutic option to treat retroperitoneal cystic lymphangioma is complete laparoscopic excision..$^{5}$ The important issue is the complete resection of the tumor with the whole adjacent organs if the surrounded tissues are involved. This would reduce the risk of recurrence from $50 \%$ to $7 \% .^{2}$ In our case, a laparoscopy was performed and upon the surgery, the adhesion of the mass to the adjacent organs was removed successfully.

\section{CONFLICT OF INTEREST}

The authors declare no conflict of interest related to this work.

\section{REFERENCES}

1. Bhavsar T, Saeed-Vafa D, Harbison S, Inniss S. Retroperitoneal cystic lymphangioma in an adult: a case report and review of the literature. World J Gastrointest Pathophysiol 2010;1:171-6. doi: 10.4291/wjgp.v1.i5.171.

2. Di Marco M, Grassi E, Vecchiarelli S, Durante S, Macchini M, Biasco G. Retroperitoneal lymphangioma: A report of 2 cases and a review of the literature regarding the differential diagnoses of retroperitoneal cystic masses. Oncol Lett 2016;11:3161-6. doi: 10.3892/ol.2016.4367.

3. Tsukamoto T, Tanaka S, Yamamoto T, Kakinoki E, Uemichi A, Kubo S, et al. Laparoscopic excision of a retroperitoneal cystic lymphangioma: report of a case. SurgToday 2003;33:142-4. doi: 10.1007/s005950300032.

4. Hauser H, Mischinger HJ, Beham A, Berger A, Cerwenka H, Razmara J, et al. Cystic retroperitoneal lymphangiomas in adults. Eur J Surg Oncol 1997;23:322-6. doi: 10.1016/S0748-7983(97)90777-0.

5. Fanaei S, Ziaee S. Retroperitoneal Cystic Lymphangioma: Case Report. Surg Sci 2011;2:209. doi: 10.4236/ss.2011.24046. 